\title{
STUDY ON THE CORRECT TILT OF THE NAVIGATION OF AGRICULTURAL MACHINERY BASED ON GPS
}

\author{
Hu Lei ${ }^{1}$, Zhou Zhifeng ${ }^{2}$ \\ ${ }^{1}$ College of Mechanical Engineering, Shanghai University of Engineering Science, Shanghai, China \\ ${ }^{2}$ College of Mechanical Engineering, Shanghai University of Engineering Science, Shanghai, China
}

\begin{abstract}
In recent years, the development of GPS navigation technology is very fast, has been applied to almost everywhere in life, especially in the field of agricultural production in recent years is more important. In foreign countries, GPS navigation application in agricultural production development has been very mature, but in China, the application of GPS in agricultural machinery navigation is still in the stage of research and development, application in agricultural machinery navigation, the GPS navigation technology to achieve accurate positioning: the most important thing is to tilt, lateral posture correction, pure line operation and a series of work based on path tracking. The GPS carrier phase measurement technology, static positioning can not only realize agricultural accurate and real-time dynamic positioning; but also can achieve high-precision attitude measurement based on observation of carrier phase receiver. Through the vector in the process of operation because of the navigation error of surface height fluctuation generated by the tilt correction algorithm, can be derived on the agricultural machinery navigation correction, because in the process of agricultural navigation operation, positioning error vector produced by the inclination will directly affect the pure path tracking algorithm based on the trajectory of the straight line thus, effects of mechanization and efficiency of intelligent agricultural production, so the research of calibration for the error caused by the tilt is of great significance. Considering the difficulty of the experiment, our research is a double side antenna attitude, so in the original location of the next step is to collect model cars some simulation of agricultural operations process data, and then use MATLAB to carry out simulation on the PC machine, according to before and after correction for the navigation of agricultural machinery industry to observe the trajectory correction effect. .
\end{abstract}

Keywords: GPS positioning; attitude measurement; tilt correction; simulation

\section{INTRODUCTION}

In this paper, the main principle of GPS navigation, carrier phase attitude side principle, finally through the analysis of the positioning error due to body tilt generated, correction algorithm is derived, to verify the effectiveness of the approach by MATLAB simulation.

\section{THE PRINCIPLE OF GPS POSITIONING}

\subsection{The Principle of Absolute Positioning}

Absolute positioning, also known as single point positioning, this is a kind of positioning using a receiver mode, it is determined by the absolute coordinates of the receiver antenna.

\subsection{The Principle of Relative Positioning}

Relative positioning, also known as differential positioning, the positioning mode uses the receiver of more than two units, at the same time were observed on the same set of satellite receiver, dynamic GPS observation of their value and DGPS data from the reference to the receiver, you can eliminate various errors, calculate the 3D coordinates of the user accurately, to determine the mutual relationship between the position of the receiver antenna. GPS relative positioning, measurement of the position is relative to a known point position, but not the absolute position in the WGS-84 coordinates of.

\section{THE PRINCIPLE OF CARRIER PHASE}

\section{MEASUREMENT}

\subsection{The Basic Principle of GPS Carrier Phase}

\section{Measurement}

Carrier phase measurement difference carrier phase is defined as the reference satellite carrier phase and receiver from observation and tracking to produce

$$
\emptyset^{j}(\mathrm{tr})=\emptyset^{j}(\mathrm{ts})-\emptyset(\mathrm{tr})
$$

In the formula: $\Phi^{j}(t s)$ for the $\mathrm{j}$ satellites carrier phase in ts emission; $\emptyset(\mathrm{tr})$ reference carrier phase generated for GPS receiver in tr

\subsection{Integer Ambiguity and Phase Cycle Slip} Problem is the Carrier Phase Measurement and Data Processing of the Two Major Problems

The carrier signal is a periodic sinusoidal signal, fractional part of the actual phase measurement can only measure less than a week, so the existence of $\mathrm{N}$ to solve the ambiguity problem, of the existence of such multiple values, is only the beginning of the carrier week count because when the receiver only from the satellite tracking is effective. If can 
get the initial $\mathrm{N}$, then to the high precision carrier wavelength to determine the path length of the satellite and the user receiver antenna solution or multiple antenna baseline attitude.

In addition, the carrier phase measurement, must continuously track the carrier, but due to the influence of receiver fault or external interference and other factors, will often lead to tracking temporarily interrupted, which will produce the phase jump of the week.

\section{THE APPLICATION OF CARRIER PHASE} MEASUREMENT FOR DETERMINATION OF ATTITUDE

\subsection{Three Coordinates Measuring the Pose of GPS}

The 3D pose parameters, short motion carrier in the threedimensional orientation parameters relative to the global or local level coordinate system -- vector coordinate system in the special coordinate system, the moving vehicle yaw angle $\alpha_{\text {yaw }}$ and lateral rolling angle $\alpha_{\text {pit }}$ and pitching angle $\alpha_{\text {rol }}$.

GPS attitude measurement means that GPS signal receiving antenna are respectively arranged in several different positions of movement carrier, using GPS carrier phase measurement to calculate 3D attitude parameters of moving vehicle. Plane GPS attitude measurement platform, determined only by the 3 points or parallel vector, therefore, at least on the moving carrier to install three GPS signal receiving antenna, in order to form two independent baselines, and the implementation of GPS attitude measurement. However, GPS is measured by WGS-84 geodetic coordinate system, and its observation value transform to the carrier coordinates and local level coordinate system.

\subsection{The Transformation between the Three}

\section{Coordinate Systems of the Gps Side Posture}

Through the definition of three kinds of coordinate system, coordinate transformation relation between can be derived WGS-84 and local level coordinate transformation can be derived; the relationship between the local level coordinate system and vector coordinate system. Through down can be found between them transform is accomplished through an orthogonal matrix. GPS is 3 for measuring the pose of the attitude angle. Because the antenna baseline coordinate components in carrier coordinate system, and the length of the baseline in the initialization phase can be accurately measured, and treat it as a known value. Therefore, as long as the real-time measured antenna baseline at the local level coordinate system in the coordinates, we can according to the local level between the coordinates and the coordinates of the vector formula for solving the corresponding attitude angle.

\subsubsection{Coordinate Transformation Relation between} WGS-84 and the Local Level Coordinate

$$
\begin{gathered}
X^{L L S}=R_{X}\left(180^{\circ}\right) R_{Y}\left(\mathrm{~B}+270^{\circ}\right) R_{Z}\left(180^{\circ}+\mathrm{L}\right) *( \\
\left.X^{\text {WGS-84 }}-X_{0}^{\text {WGS-84 }}\right)
\end{gathered}
$$

Type: $\mathrm{X}_{0}^{\mathrm{WGS}-\mathrm{E4}}$ as the station coordinates, In the process of solving the attitude measurement, it is mainly measured by the antenna point coordinates (LLS axis).

4.2.2 The Transformation between the Local Level Coordinate System and Vector Coordinate System

$$
X^{B F S}=R_{z}\left(\alpha_{\text {rol }}\right) R_{y}\left(\alpha_{\text {pit }}\right) R_{z}\left(\alpha_{\text {yaw }}\right) X^{L L S}
$$

\section{DOUBLE SIDE ANTENNA ATTITUDES}

\subsection{The General Principle of Application of Carrier}

\section{Phase Measurement}

GPS is a dual antenna attitude measurement at two different positions of a moving carrier are respectively installed GPS signal receiving antenna, using GPS carrier phase measurement to calculate two-dimensional attitude parameters of moving vehicle. The motion vector using two antenna GPS attitude determination, can only estimate the attitude angle two.

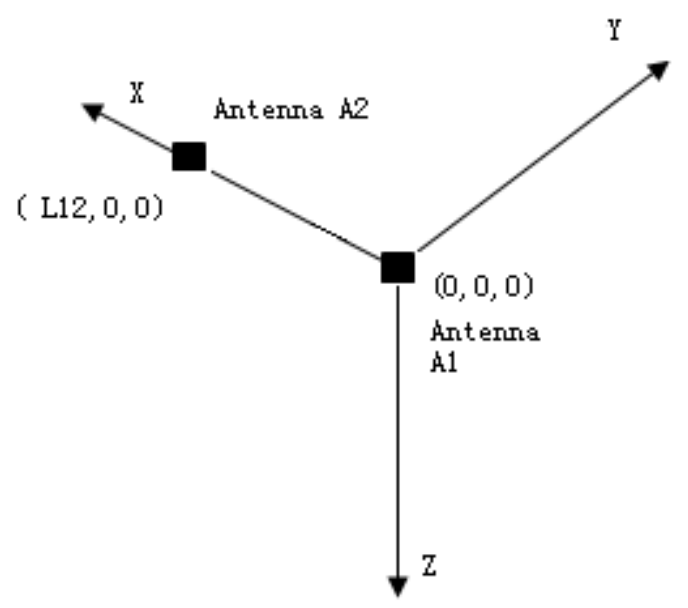

Fig-1: Two antenna schematic diagram in the vector coordinate system

For the antenna configuration on a rigid body, the distance between the antennas can be accurately measured, and the motion state remains constant, coordinate position of two antennas in carrier coordinate system is ensured. Figure 2 shows the L12 is the length A12 baseline. Vector directional antenna A1 to A2 antenna can determine the yaw angle, coordinate antenna A2 $(\mathrm{L} 12,0,0)$, the A1 is set to the origin of the antenna carrier coordinates and local level coordinate 
system, can directly calculate the yaw angle $\alpha_{\text {yaw }}$ and pitching angle $\alpha_{\text {pit: }}$ :

$$
\begin{gathered}
\alpha_{\text {yaw }}=-\tan ^{-1}\left(\frac{y_{2}^{L L S}}{x_{\mathrm{z}}^{L L S}}\right) \\
\alpha_{\text {pit }}=-\tan ^{-1}\left(\frac{z_{\mathrm{z}}^{L L S}}{\sqrt{\left(x_{\mathrm{z}}^{L L S}\right)^{2}+\left(y_{2}^{L L S}\right)^{\mathrm{z}}}}\right)
\end{gathered}
$$

Through the observation of the GPS carrier phase, the 3D position can be extremely accurately determination of relative on antenna $\mathrm{A} 2$ and $\mathrm{A} 1$ in WGS-84 geocentric coordinate system, namely the projection in the geocentric coordinate system in the baseline, according to the conversion of WGS-84 coordinates to LLS (local level system) formula of coordinate is transformed into coordinates in antenna A1 for the origin of the local level coordinate system, and then according to the above formula (4) and (5) can calculate the yaw angle and pitching angle.

\subsection{The Individual Difference in Attitude}

\section{Measurement and Double Difference}

GPS attitude determination using carrier phase observation method, the instantaneous phase of GPS carrier signal measurement of both ends of the baseline antenna receiving difference, vector direction method for solving the phase difference of the baseline, to determine moving body posture; pseudo range method is essentially a method of phase measurement. The definition of baseline $A B$ in satellite line of sight on the projection of up to $\Delta \emptyset 1$ for the carrier phase difference between antenna A and B

$$
\Delta \emptyset_{1}=N+n
$$

$\mathrm{N}$ is the number of integer cycle, $\mathrm{n}$ is cycle phase fraction. Because of in the actual measurement, the instantaneous phase cannot be measured directly, can only be measured between satellite carrier signal and receiver reference signal phase difference, i.e.

$$
\emptyset_{1}^{T}=\emptyset_{s}^{T}-\emptyset_{n}^{T}+N_{b}-\varepsilon
$$

Represents the phase satellite at time $T, \emptyset_{\mathrm{n}}^{\mathrm{T}}$ is the receiver phase, $\mathrm{N}_{\mathrm{b}}$ Called ambiguity, $\varepsilon$ is the measurement error. Then, the carrier phase difference measurement:

$$
\begin{aligned}
& \Delta \emptyset_{1}=\Delta \emptyset_{1 A}^{T}-\Delta \emptyset_{1 B}^{T}=\left(\emptyset_{n A}^{T}-\emptyset_{n B}^{T}\right)+\left(N_{b}^{A}-\right. \\
& \left.N_{b}^{B}\right)
\end{aligned}
$$

Make: $\emptyset_{n A}^{T}-\emptyset_{n B}^{T}=\mathrm{n}, N_{b}^{A}-N_{b}^{B}=N$.

The single carrier phase difference can not only eliminate the ionosphere, troposphere delay error, but also can eliminate the error of satellite orbit. Further, in order to eliminate the receiver clock error, can also be used for double difference, double difference is obtained in individual difference equation is selected as a main satellite, two main antenna for satellite and other different satellites were obtained with single difference observations and solve the difference, i.e.

$$
\Delta \emptyset_{2}=\Delta \emptyset_{1}^{S 1}-\Delta \emptyset_{1}^{S 2}
$$

$\Delta \emptyset_{2}$ Known as the double difference, It eliminates the receiver clock error and the machine initial phase error, but there is still ambiguity problem, if ambiguity, can get the solution of $\mathrm{AB}$ baseline vector through the least squares algorithm.

\section{TILT CORRECTION OF GPS POSITIONING}

\section{ERROR IN THE NAVIGATION OF} AGRICULTURAL MACHINERY

\subsection{GPS Navigation System Is An Important Equipment Of Precision Agriculture}

Its accuracy directly affects the precision of navigation. In the field of agricultural machinery driving, affected by the surface fluctuation effect of car will have a certain degree of tilt, as shown in figure 3

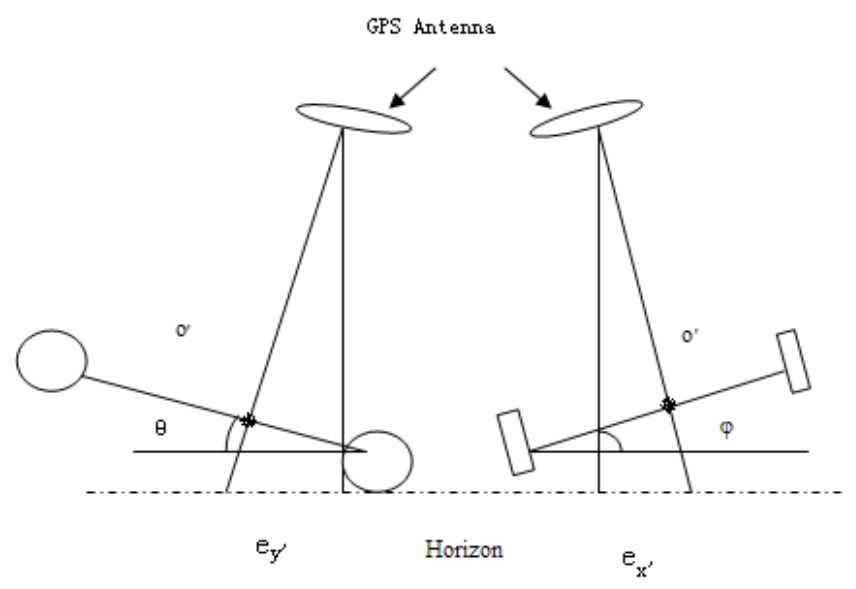

Fig-2: model car pavement impact attitude angle change

Note: $\theta$ represents the pitch produced by the inclination angle before and after; $\varphi$ represents the roll angle of tilt.

In the navigation of agricultural machinery in the control process, the main use of two coordinate systems, namely the local level coordinate system and body coordinate system, as shown in figure 4. The local level coordinate system is represented by a diagram of the $\mathrm{x}$ o $\mathrm{y}$ coordinate system. The vehicle coordinate system defined as the vehicle body centroid coordinates origin, the body direction as y axis, the right side of the vertical direction as $\mathrm{x}$ axis, represented by a diagram of the $x^{a} \quad o^{a} \quad y^{a}$ coordinate system. Figure 4 the parameter specified for the current vehicle heading angle. 


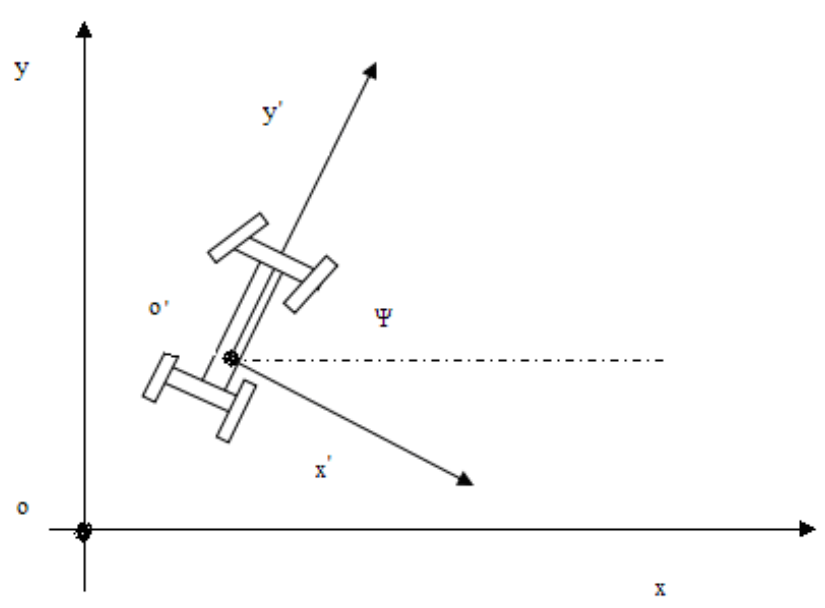

Fig-3: the above $x$ o y on behalf of the local level coordinate system; $x^{n} \quad a^{n} \quad y^{a}$ represents body coordinate system; $\psi$ represent the heading angle.

For the correction of GPS positioning error caused by the body tilt, first calculate positioning errors and error of the positioning in the longitudinal direction of vehicle tilt in the horizontal direction in the body coordinate system:

$$
\begin{array}{r}
\mathrm{e}_{x^{0}}=H \sin (\varphi) \\
e_{y^{0}}=H \sin (-\theta)
\end{array}
$$

Type $H$ means Vehicle antenna height of apart from the ground.

The projection of $e_{\mathrm{M}}^{\mathrm{e}}$ and $\mathrm{e}_{\mathrm{y}}$ transformation to the local level coordinate system, get the car body tilting position error produced in the horizontal direction and the error of position in the longitudinal direction:

$$
\begin{array}{r}
e_{x}=\mathrm{e}_{x^{p}} \sin \psi+e_{y^{\prime}} \cos \psi \\
e_{y}=-\mathrm{e}_{x^{p}} \cos \psi+e_{y^{\prime}} \sin \psi
\end{array}
$$

Assume that $\left(\mathrm{x}_{\mathrm{ak}} \mathrm{Y}_{\mathrm{ak}}\right)$ to the original location level model car at $\mathrm{k}$ coordinates, $\left(\mathrm{x}_{\mathrm{blk}}, \mathrm{y}_{\mathrm{bk}}\right)$ for positioning after the tilt correction $\mathrm{K}$ model car time coordinates, can be derived from the relationship between the two is:

$$
\begin{aligned}
& x_{b k}=x_{a k}+\mathrm{e}_{x^{0}} \sin \psi_{k}+e_{y^{\prime}} \cos \psi_{k} \\
& y_{b k}=y_{a k}-\mathrm{e}_{x^{\prime}} \cos \psi_{k}+e_{y^{\prime}} \sin \psi_{k}
\end{aligned}
$$

In the formula: $\psi_{\kappa}$ is the heading angle for body at $\mathrm{k}$ time.
7. THE SIMULATION EXPERIMENT BASED ON THE MATLAB COLLECTION OF THE ORIGINAL NAVIGATION DATA

7.1 Model Cars Expected Drive Trajectory When the Baseline is Formed

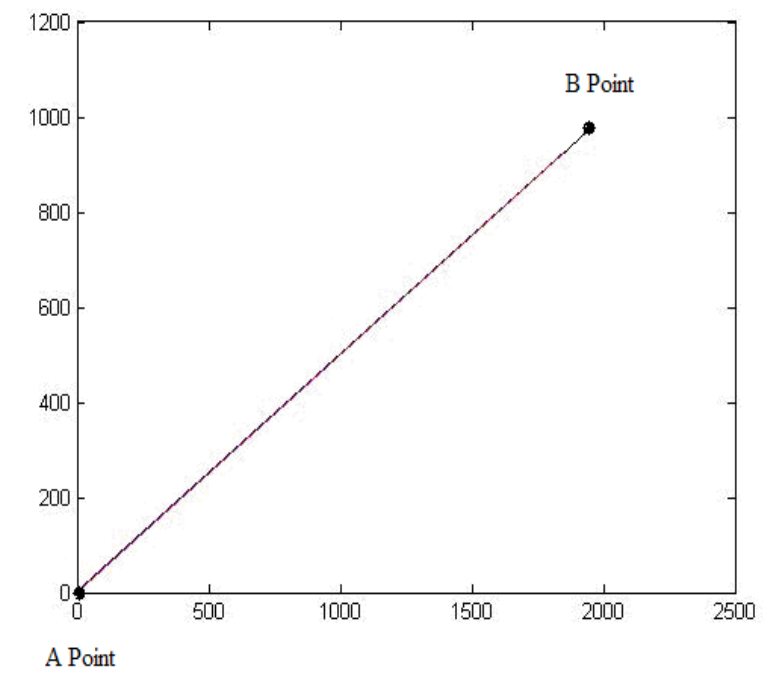

Fig-4: the horizontal axis represents positive direction of the local level coordinate system, the angle between the baseline $\mathrm{AB}$ and the horizontal axis is the initial heading angle, the horizontal axis and vertical axis units are meters.

\subsection{Simulation of the Traveling Path of the Model}

\section{Car before joining the tilt Correction Algorithm}

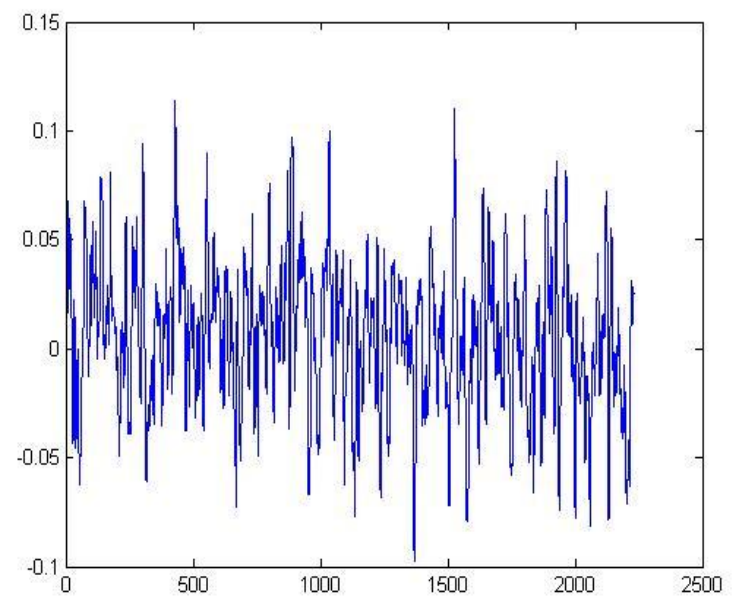

Fig-5: the simulation chart shows the horizontal error size between the car real driving trajectory and the baseline $A B$ in the test process without addition of the tilt calibration algorithm. 


\subsection{Simulation of the Traveling Path of the Model}

Vehicle after the Addition of the Tilt Correction

\section{Algorithm}

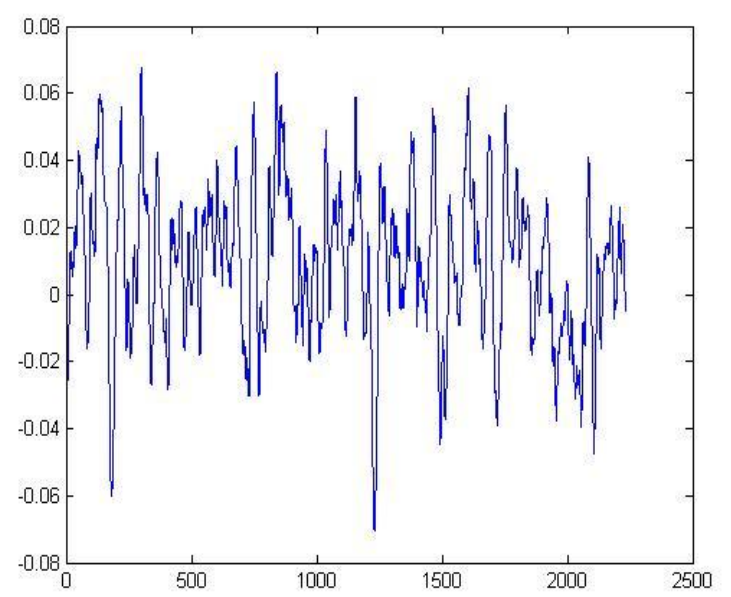

Fig-6: the simulation chart shows the horizontal error size between the car real driving trajectory and the baseline $\mathrm{AB}$ in the test process with addition of the tilt calibration algorithm.

\section{CONCLUSION}

By comparison, finding GPS navigation model car is closer to the ideal expected path than no correction to the running track after joining the tilt correction algorithm. So in the work of agricultural machinery navigation, the positioning error caused by the body tilt calibration is necessary, the agricultural machinery production efficiency has been improved, significant.

\section{ACKNOWLEDGEMENTS}

The author would like to thank Professor Zhou Zhifeng who helped and supported with this work. This work was supported by Shanghai University of Engineering Science postgraduate innovation project(14KY0131)

\section{REFERENCES}

[1]. Hatch R. Ambiguity Resolution in the Fast Lane [R]. ION GPS-89, Colorado, Sept, 1989:45-50

[2]. Elliott D K. Understanding GPS Principles and Applications. Artech1 House.1996

[3]. Hatch R. Ambiguity Resolution in the Fast Lane [R]. ION GPS-89, Colorado, Sept, 1989:45-50

[4]. Frank Van Grass, Michael Bra. GPS Interferometric Attitude and Heading Determination: Initial Flight Test Results. GPS World, 1991, 38(4):297-316

[5]. Liu Yu. GPS Carrier phase measurement positioning theory. Navigation, 1994, 03: 52-63

[6]. Hatch, R. Ambiguity Resolution in the Fast Lane, Proceedings of the Second International Technical Meeting of the Satellite Division of the ION, GPS-89, Colorado Springs, CO, September 26-29, 1989.
[7]. Wolf, R., Hein, G W., Eissfeller1, B., etc., An Integrated Low-cost GPS/INS Attitude Determination and Position Location System, in Proceedings of ION GPS-96, September 17-20, 1996: 975-981: Kansas City, Missouri. [8]. Mathur1, N.G., A New Coning Attitude Algorithm for a Low Cost INS-GPS Integration, in Proceedings of ION GPS-1997, September 16-19, 1997: 807-814: Kansas City, Missouri.

[9]. Brown Ronald A. Instantaneous GPS Attitude Determination .Proceeding of IEEE Position Location and Navigation Symposium ,PLANS'92,1992:113-120

[10]. Remo B. W. Pseudo-kinematic GPS results using the ambiguity functions method. Navigation, 1991, 38(1), 1736.

\section{BIOGRAPHIES}

Hu Lei: Reading of postgraduate, College of Mechanical Engineering, Shanghai University of Engineering Science. The main research filed for the computer and control, intelligent agricultural equipment.

Telephone: 15317860239

Email:871258250@qq.com

Zhou Zhifeng: College of Mechanical Engineer, Shanghai University of Engineering Science, PhD, associate professor. Main research filed for the computer measurement and control equipment, signal processing. E-mail:zhousitu@126.com 